\title{
"Biología, ecología y manejo de Monochamus galloprovincialis (Olivier), vector del nematodo de la madera del pino"
}

\author{
Sanchez-Husillos, $\mathrm{E}^{1^{*}}$., Etxebeste, I., ${ }^{1}, \&$ Pajares, J. ${ }^{1}$ \\ ${ }^{1}$ Instituto de Investigación Forestal Sostenible, \\ U. Valladolid-CIFOR - INIA. Palencia, España \\ *Autor para correspondencia: estela.husillos@gmail.com
}

\section{Introducción}

La actividad humana ha estado muy ligada al movimiento ya sea intencionado o fortuito de organismos vivos exóticos que ponen en serio peligro nuestros ecosistemas. Un importante caso que afecta a nuestros bosques fue la introducción del nematodo del pino Bursaphelenchus xylophilus (Steiner and Buhrer) Nickle (Nematoda: Parasitaphelenchidae) causante de la más importante enfermedad que está destruyendo actualmente nuestros pinares: "la enfermedad del marchitamiento del pino".

Este organismo originario de América fue accidentalmente introducido en Asia en 1905 (Yano, 1913) donde ha causado daños devastadores en sus pinares originando pérdidas millonarias (Yoshimura et al., 1999). En 1998 fue detectado por primera vez en Europa en la península de Setúbal, Portugal (Mota et al., 1999). Los primeros estudios demostraron que el único vector en Portugal es el perforador de los pinos Monochamus galloprovincialis Olivier (Coleoptera, Cerambycidae), insecto que se encuentra distribuido por toda Europa, Cáucaso, Siberia, Mongolia, China y el norte de África. Ambos, B. xylophilus y sus vectores, se clasificaron como un organismo de cuarentena de la clase "A-1" (OEPP / EPPO 1986). 
Desde la detección del primer positivo en Europa, Portugal implementó un programa de erradicación en las zonas demarcadas. Sin embargo, a pesar de los intensos esfuerzos, la enfermedad sigue dispersándose por todo Portugal. Debido a esta dramática situación, un plan de contingencia oficial contra la enfermedad encabezado por el Ministerio de Medio Ambiente y de acuerdo con el Comité Fitosanitario Permanente de la Unión Europea, puso en marcha medidas preventivas en 2008 coincidiendo con el primer positivo en España en una zona cercana a la frontera con Portugal. Desde entonces, un sistemático muestreo a lo largo de toda la frontera portuguesa ha detectado la presencia de hasta cinco positivos que están en proceso de erradicación, siempre en zonas cercanas a Portugal (Abelleira et al., 2011; Zamora et al., 2015). Esto va a indicar el claro riesgo de la difusión de la enfermedad dentro del territorio español por medio de Portugal. La última decisión de la Comisión de la Unión Europea relativa a la prevención de la propagación de la enfermedad (2012/535/UE) incluía medidas de erradicación obligatorias que forzaban a realizar cortas a hecho de al menos $500 \mathrm{~m}$ alrededor de cada planta sensible en la que se había detectado la enfermedad. Esto va a suponer la corta de miles de hectáreas en nuestro país si se detecta un positivo. Pero todas estas medidas de contención y erradicación se van a basar en las experiencias previas de los países asiáticos donde sus vectores se van a dispersar en cortas distancias concentrándose la mayoría en distancias muy próximas a su lugar de origen. Sin embargo, esto no tiene por qué significar que el vector europeo se comporte igual, como ya fue observado por Mas et al. (2015), capturando insectos dispersados hasta $16 \mathrm{~km}$ de su origen. Debido a estas imperfecciones del plan es necesario profundizar en el conocimiento de $M$. galloprovincialis para diseñar un plan de contingencia apropiado y así poder desarrollar una eficaz estrategia de manejo y erradicación de la enfermedad.

\section{Antecedentes}

Desde su introducción en los países asiáticos, el manejo de la enfermedad a través de sus insectos vectores ha sido considerado como una de las estrategias más prometedoras. Sin embargo, los antecedentes en el estudio de Monochamus galloprovincialis en Europa son prácticamente inexistentes hasta la aparición del nematodo del pino en Portugal, y la demostración de la estrecha asociación entre ambos organismos (Sousa et al., 2001), por lo que se podría decir que ha sido en la última década cuando se ha despertado el interés por este insecto, más si cabe por la detección del nematodo también en España.

Hasta estudios recientes no se disponía de un sistema de captura operativo que fuese efectivo, y de ahí la inseguridad de los conocimientos que disponíamos de este insecto debido a la incapacidad de capturar esta especie en campo (Shibata, 1986; Togashi, 1990; Robinet et al., 2009). En los últimos años se han identificado atrayentes eficaces en campo para $M$. galloprovincialis, entre los que se encuentra su feromona de agregación, cairomonas de los pinos hospedantes como el $\alpha$-pineno, y cairomonas de escolítidos como el ipsenol y el 2-metil-3-buten-2-ol (Pajares Alonso et 
al., 2004, 2010; Ibeas et al., 2007), mientras que a su vez se han desarrollado trampas altamente eficaces gracias a la incorporación del teflón y el embudo colector alargado que van a permitir la captura de los insectos vivos (Alvarez et al., 2015). Estos avances han permitido el desarrollo de un dispositivo de captura altamente eficaz que va a permitir estudiar su biología y ecología, y así desarrollar técnicas que nos van a permitir elaborar una estrategia de control y manejo de la enfermedad adecuada. Estos resultados van a ser de gran utilidad para la obtención de información práctica que permita al Comité Fitosanitario Permanente de la Unión Europea elaborar políticas científicas y sanitarias respecto al problema del nematodo del pino y elevarlas a la Comisión para que sean incorporadas como decisiones vinculantes.

\section{3. ¿Cuáles son los hospedantes de Monochamus galloprovincialis en la Península?}

Se realizaron diversos bioensayos para conocer el espectro de hospedantes del insecto en la Península Ibérica, tomando como protagonista el piñonero debido a resultados previos obtenidos en Portugal donde esta especie fue rechazada en laboratorio, además de observar cómo en las áreas afectadas donde el piñonero se encontraba de forma dispersa, no fue afectado por la enfermedad (Naves et al., 2006). Para conocer los escenarios de mayor riesgo potencial de dispersión de la enfermedad se sometió al insecto a bioensayos de doble elección en los que se evaluó la preferencia de hospedantes tanto de alimentación como oviposición finalizando con ensayos de supervivencia del insecto en Pinus pinea L.

Se obtuvo una escala de preferencias de hospedantes destacando Pinus sylvestris L. como primer candidato seguido de $P$. pinaster. Por otro lado, se observó cómo el insecto no sólo no rechaza el piñonero sino que, a pesar de no ser la especie preferida, fue capaz de alimentarse, reproducirse y desarrollar su progenie en dicha especie como contraposición a los resultados obtenidos en Portugal (Naves et al., 2006). En cambio, se obtuvo un posible efecto disuasorio del limoneno, principal terpeno del piñonero, que podría explicar de alguna manera esa no preferencia hacia este hospedante y abrir una posible puerta para el manejo del insecto.

\section{4. ¿Nacen con musculatura y lípidos suficientes para emprender vuelo? ¿Cuánto podrían dispersarse los inmaduros?}

En los monitoreos de campo se observó cómo insectos recién emergidos no eran capturados hasta pasadas 2 semanas, lo que supone un freno para el conocimiento del comportamiento de dispersión durante este período clave para la contención de la enfermedad. Este período de no atracción por el cebo podría atender a alguna razón fisiológica del insecto y podría incluso afectar a su capacidad de vuelo, por lo que se procedió a analizar el desarrollo gonádico de insectos de ambos sexos en intervalos comprendidos entre recién emergidos y 21 días. Así, se obtuvo una maduración se- 
xual en un período comprendido entre los 11-12 días para ambos sexos (Fig. 1) que va a coincidir con ese período de no atracción por el cebo. Además se realizaron bioensayos consistentes en la extracción de la musculatura alar y contenido en lípidos de insectos de ambos sexos en esos mismos intervalos y se les escaneó mediante microtomografía computarizada de rayos- $\mathrm{X}$ a alta resolución para poder observar sus cambios fisiológicos, así como las estructuras de su musculatura alar (Fig. 2). Se estimó que los insectos nacen con musculatura y reservas suficientes como para emprender vuelo, resultando además que no varían a lo largo de la edad y sin afectarles el tamaño o sexo. Como paso final se realizó un ensayo en campo para testar esa capacidad de dispersión de insectos recién emergidos. Se liberaron 500 individuos recién emergidos en un área desprovista de posibles hospedantes a diferentes distancias comprendidas entre $100-3000 \mathrm{~m}$, capturándose en una pequeña masa de $P$. pinaster mediante trampas multiembudos cebadas. El ajuste de un modelo negativo exponencial indicó que los insectos inmaduros pudieron dispersarse hasta los $3109 \mathrm{~m}$ desde el punto de liberación atravesando campos de cultivo hasta un pequeño rodal de pinos. A su vez se obtuvo una supervivencia media de 12 días en insectos sometidos a ayuno en laboratorio, datos que en su conjunto ponen de manifiesto la necesidad de reforzar las medidas de detección en procesos de transporte de madera y controles en puertos y demás entradas de posible material infectado.

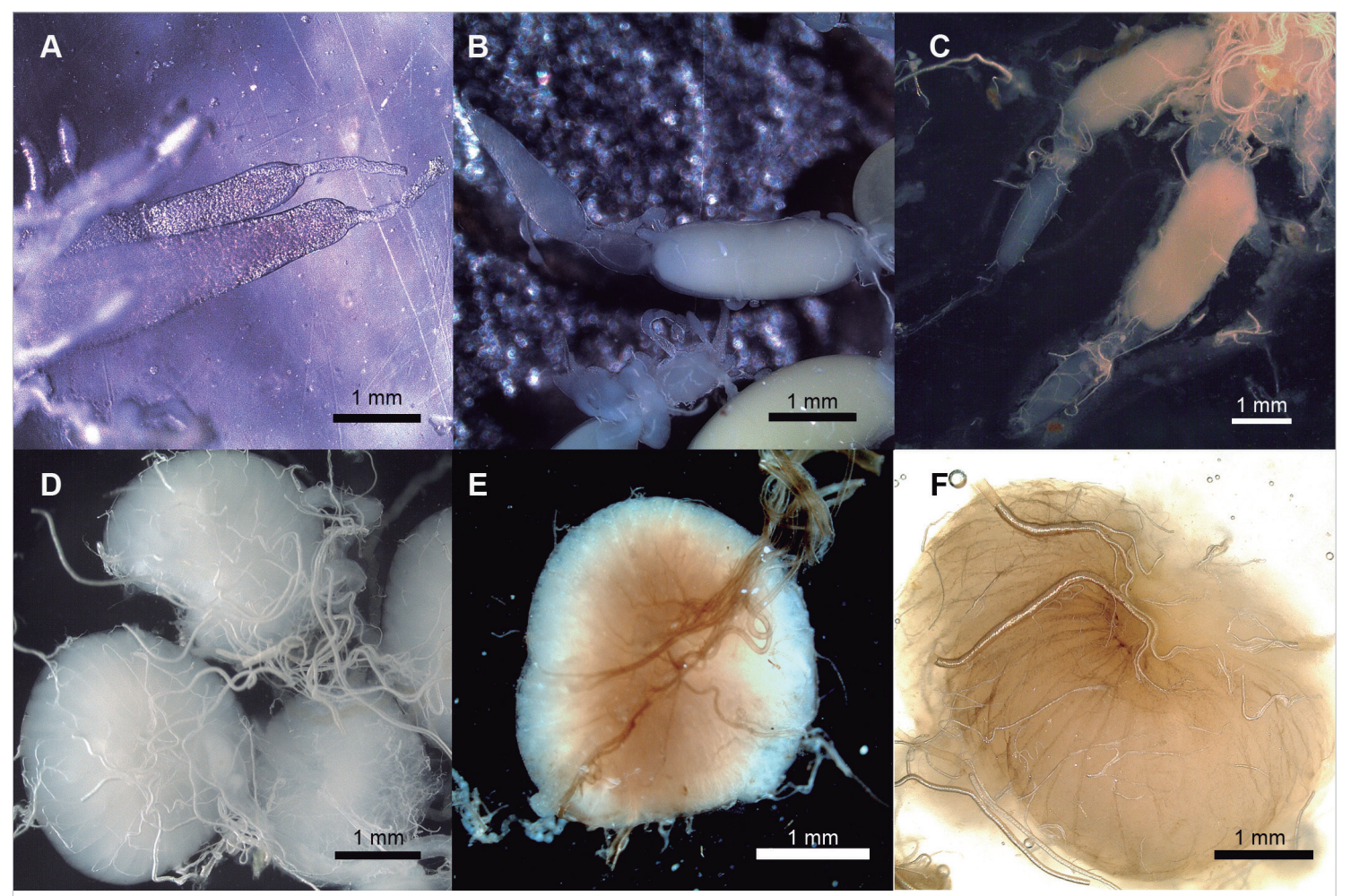

Figura 1. Desarrollo gonádico en M. galloprovincialis después de 0, 8 y 14 días de alimentación desde su emergencia. Las figuras a, b y c corresponderían al desarrollo de los ovariolos mientras que d, e y f, el cambio de coloración en los testículos masculinos. 


\section{5. ¿Cómo es la dispersión de los adultos de Monochamus galloprovincialis?}

La enfermedad del nematodo del pino se ha extendido accidentalmente a escala mundial principalmente por el transporte humano. Sin embargo, a escala local la dinámica de colonización será determinada por el comportamiento de dispersión de los insectos. Este último parámetro va a tener su importancia a la hora de diseñar los protocolos de contención de la enfermedad.

Para estudiar esta dispersión se realizaron 3 ensayos en campo usando técnicas de captura-marcado-recaptura (CMR) en diferentes escenarios de masas contínuas y fragmentadas. Para ello se liberaban insectos identificados en el centro de un dispositivo formado por un grid de trampas ubicadas a diferentes distancias (Fig 3).

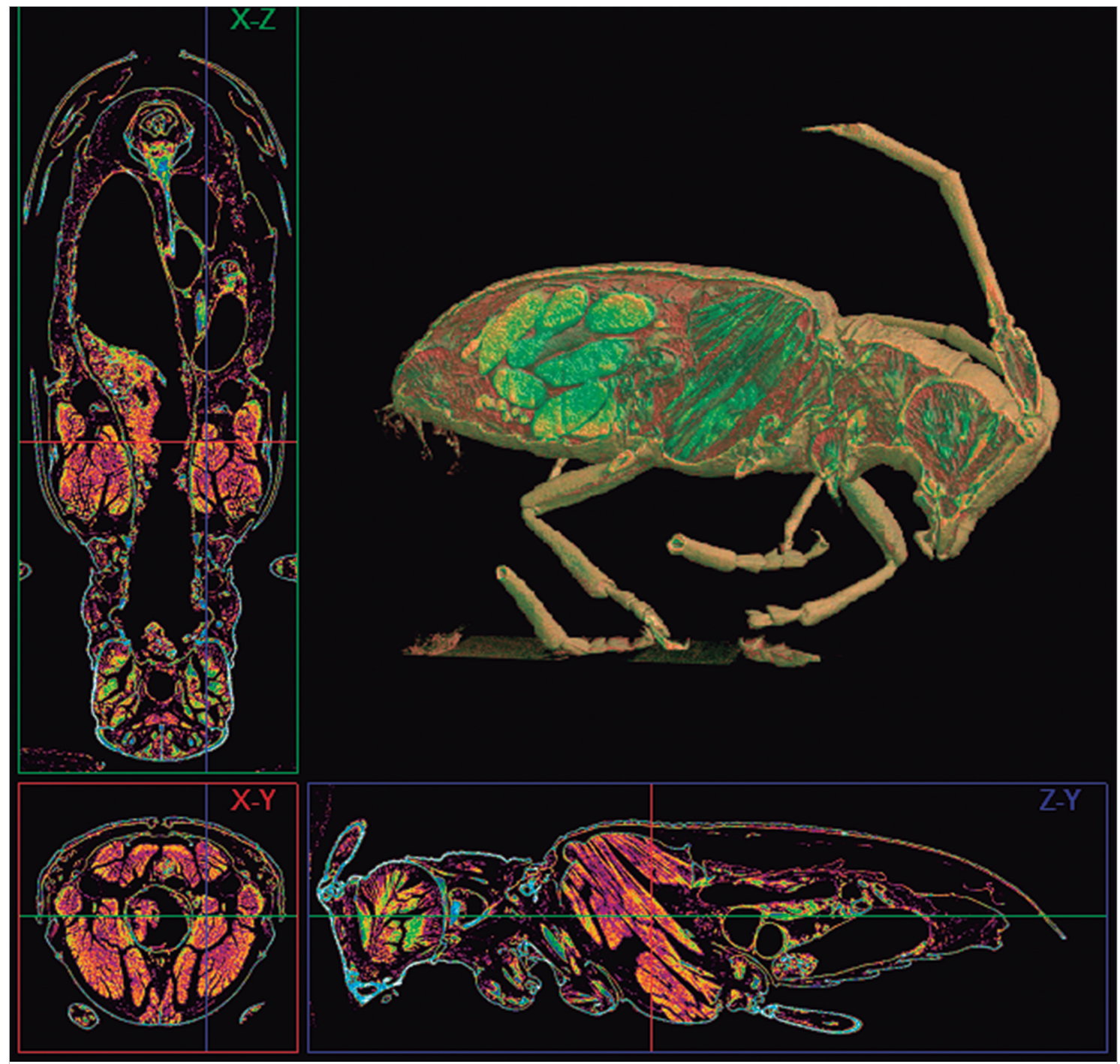

Figura 3. Reconstrucción en CTvox de una hembra madura de Monochamus galloprovincialis escaneada mediante un escáner Skyscan 1172 Micro-CT de alta resolución. 
Se obtuvo que la mayoría de los insectos se van a concentrar a distancias cercanas a su origen y el $99 \%$ alcanzará hasta los $3500 \mathrm{~m}$. Sin embargo, existen unos pocos insectos largo dispersantes que van a ser capaces de alcanzar hasta los $5 \mathrm{~km}$, distancia que aumenta a medida que se fragmenta el hábitat (Tabla 1). Esto pone en duda los protocolos implantados hasta hoy sobre la erradicación de la enfermedad respecto a la distancia de las cortas.

Tabla 1. Resumen de los resultados de estudios de marcado-captura-recaptura de Monochamus galloprovincialis entre 2009 y 2011. Representa el número de insectos liberados ( $\left.\mathrm{N}_{0}\right)$, capturas absolutas y relativas, y rangos y valores medios de distancias y tiempos de recapturas.

\begin{tabular}{|c|c|c|c|c|c|c|c|c|c|c|c|c|}
\hline \multirow[b]{2}{*}{ Año } & \multirow[b]{2}{*}{ Réplica } & \multirow[b]{2}{*}{ Fecha } & \multirow{2}{*}{\multicolumn{2}{|c|}{$\mathbf{N}_{0}$}} & \multirow{2}{*}{\multicolumn{2}{|c|}{ Recapturas }} & \multicolumn{3}{|c|}{ Distancia (m) } & \multicolumn{3}{|c|}{ Tiempo (días) } \\
\hline & & & & & & & $\mathbf{d}_{\min }$ & $\mathbf{d}_{\max }$ & $\mathbf{d}_{\text {med }}$ & $\mathbf{t}_{\min }$ & $\mathbf{t}_{\max }$ & $\mathbf{t}_{\text {med }}$ \\
\hline \multirow[t]{4}{*}{2009} & I & 2009-06-24 & 54 & & 18 & $(33.3 \%)$ & 50 & 500 & 100 & 28 & 105 & 45.5 \\
\hline & II & 2009-07-01 & 55 & $(36+19$ ठో) & & $(38.2 \%)$ & 50 & 500 & 50 & 21 & 91 & 52 \\
\hline & III & 2009-07-08 & 40 & $(19 \uparrow, 21 ठ)$ & 11 & $(27.5 \%)$ & 50 & 250 & 50 & 7 & 77 & 35 \\
\hline & IV & 2009-07-15 & 22 & (10ㅇ,12ठ) & 10 & $(45.5 \%)$ & 50 & 500 & 75 & 21 & 84 & 35 \\
\hline \multirow[t]{4}{*}{2010} & I & $2010-06-29$ & 39 & $(22+, 17$ ठ) & 9 & $(23.1 \%)$ & 71 & 762 & 141 & 14 & 49 & 14 \\
\hline & II & $2010-07-06$ & 106 & $(53+, 53$ ठ $)$ & 32 & $(30.2 \%)$ & 71 & 762 & 71 & 7 & 84 & 28 \\
\hline & III & $2010-07-13$ & 142 & $(66+76 \circlearrowright 0)$ & 43 & $(30.3 \%)$ & 71 & 762 & 141 & 14 & 84 & 28 \\
\hline & IV & $2010-07-20$ & 66 & $(34 \%, 32 \precsim)$ & 18 & $(27.3 \%)$ & 71 & 762 & 141 & 14 & 77 & 24.5 \\
\hline \multirow[t]{7}{*}{2011} & I & 2011-06-02 & 128 & (61+,67ठ) & 1 & $(0.8 \%)$ & 0 & 0 & 0 & 29 & 29 & 29 \\
\hline & II & 2011-06-10 & 81 & $(32+, 67 \delta)$ & 3 & $(1.2 \%)$ & 559 & 1000 & 901 & 52 & 94 & 73 \\
\hline & III & 2011-06-15 & 77 & $(44+, 34$ ठ) & 6 & $(1.3 \%)$ & 0 & 2019 & 0 & 26 & 89 & 75 \\
\hline & IV & 2011-06-22 & 78 & 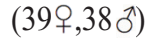 & 5 & $(1.3 \%)$ & 0 & 559 & 0 & 19 & 78 & 61 \\
\hline & V & 2011-07-04 & 58 & (40+,18ठ) & 7 & $(1.7 \%)$ & 0 & 1118 & 500 & 35 & 98 & 56 \\
\hline & VI & 2011-07-11 & 32 & $(18+, 14 ठ ో)$ & 3 & $(3.2 \%)$ & 0 & 559 & 559 & 28 & 59 & 49 \\
\hline & VII & 2011-07-21 & 19 & $(13$ ㅇ, $6 ठ)$ & 3 & $(5.3 \%)$ & 0 & 5303 & 5037 & 14 & 46 & 49 \\
\hline
\end{tabular}

\section{6. ¿Cómo se dispersa el insecto según la estructura de la masa?}

Gracias al desarrollo de las tecnologías LiDAR, podemos realizar una caracterización de la masa forestal con un nivel de descripción muy exhaustivo, lo cual va a ser muy útil para estudiar la influencia del paisaje y estructura de la masa en las dinámicas de poblaciones. De esta manera se obtuvieron 7 variables dasométricas de la masa (Fig 4): la densidad estratificada del dosel (Evans et al., 2009) el \% de cobertura hasta $5 \mathrm{~m}$ (Cover0-5), el \% de cobertura por encima de $5 \mathrm{~m}$ (Cover5) y la fracción de cabida cubierta (FCC), la altura máxima del arbolado, la altura del regenerado y la altura de la inserción de copas. Se añadió también la cuenca visual de las trampas obtenida mediante técnicas SIG. Estas variables fueron utilizadas como una aproximación para conocer la preferencia del insecto según las capturas obtenidas en campo en un ensayo de CMR en el que se había realizado la digitalización de estas variables. 


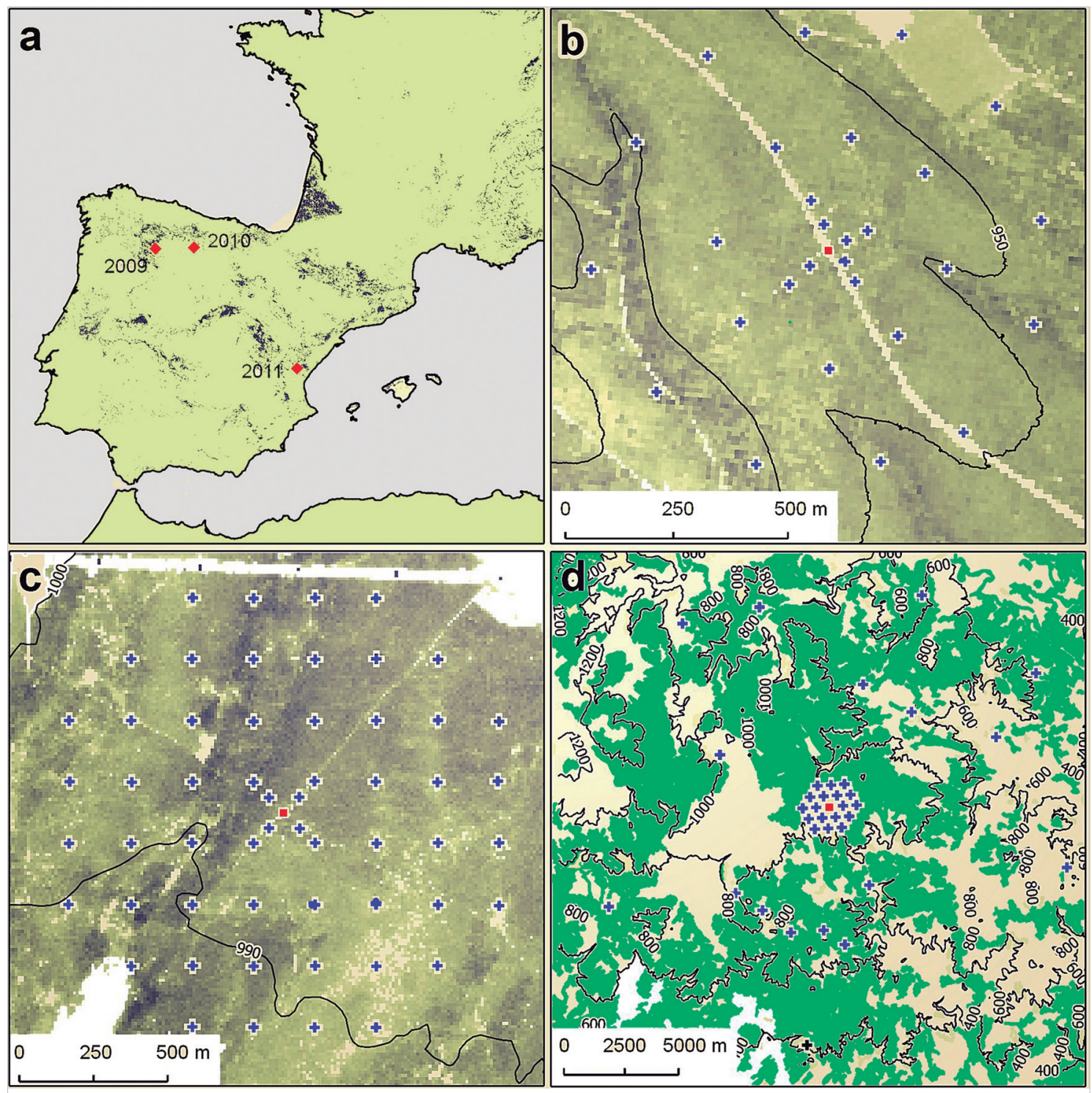

Figura 4. Localización y diseño de los dispositivos de trampeo utilizados para los ensayos de dispersión de Monochamus galloprovincialis. Las cruces azules representan la localización de las trampas y el cuadrado rojo el sitio de liberación de los insectos.

Se obtuvo que la abundancia de los insectos nativos se ve influida positivamente por la fracción de cabida cubierta, lo cual va estar correlacionado con la edad de la masa. Los insectos liberados se van a desplazar peor en zonas donde el porcentaje de cobertura menor de $5 \mathrm{~m}$ es mayor, probablemente debido al aumento de obstáculos que dificultan su vuelo.

\section{Manejo del insecto con captura masiva}

Se realizaron dos ensayos captura-marcado-recaptura para evaluar el efecto de la 

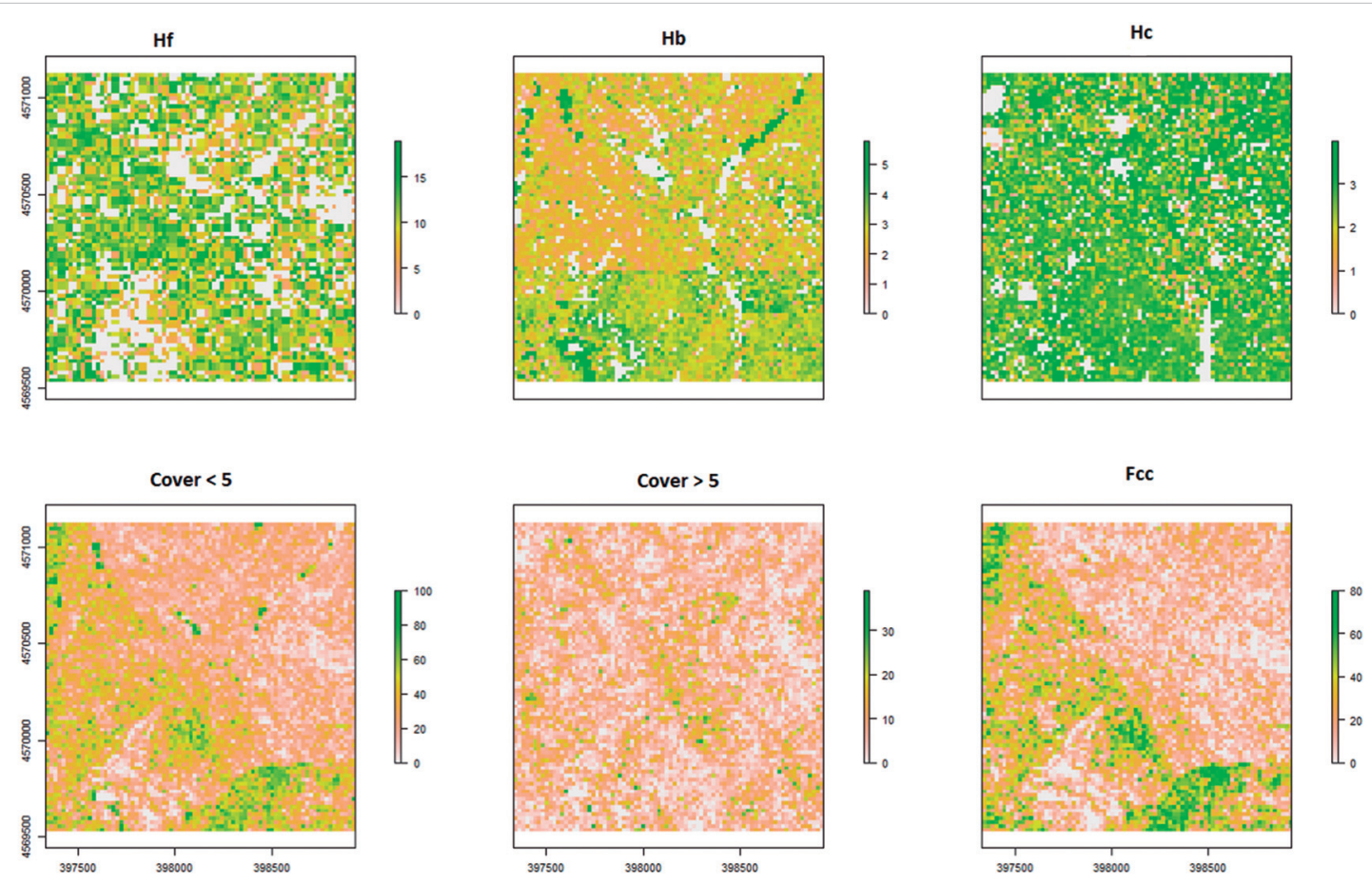

Figura 5. Variables de la masa obtenidas mediante técnicas LiDAR en la zona de muestreo.

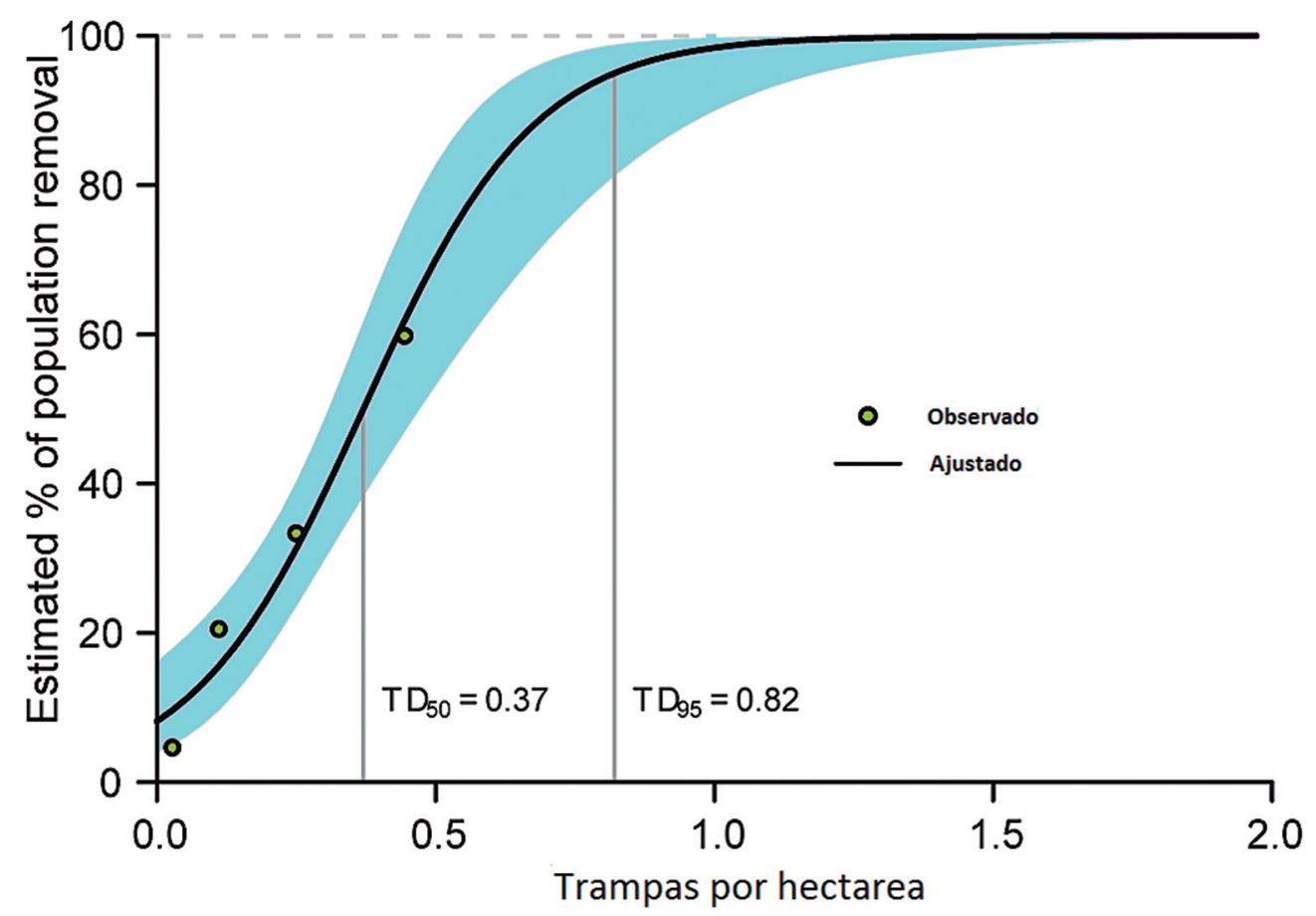

Figura 6. Ajuste de la regresión logística con las capturas en relación al incremento de la densidad de trampeo. La región sombreada representa el intervalo de confianza del $95 \%$ de las respuestas ajustadas. Los valores TD50 y TD95 van a señalar el valor de trampas por hectárea necesarios para extraer el 50 y $95 \%$ de la población respectivamente. 
captura masiva en las poblaciones de M. galloprovincialis. En el primero se evaluó un método válido para estimar la abundancia de población de este insecto consistente en la validación de los modelos Jolly-Seber para la obtención de la abundancia poblacional en poblaciones abiertas.

En el segundo se analizó la relación entre la densidad de las trampas y la cantidad de insectos extraídos, de manera que los gestores de salud de los bosques puedan elegir la densidad de captura óptima adecuada a sus objetivos (Fig. 5).

De esta manera se obtuvo la densidad poblacional de Monochamus en la zona de estudio en torno a 82 individuos/ha. Así se pudo calcular cómo intensidades de trampeo de $0.02,0.11,0.25$ y 0.44 trampas/ha extrajeron el $4.66,20.50,33.33$, y $59.80 \%$ de la población de $M$. respectivamente representando un método de gestión muy prometedor para la contención o erradicación de B. xylophilus en zonas de especial interés o bordes de masa.

\section{Bibliografía}

Abelleira A., Picoaga A., Mansilla JP \& Aguin O ., 2011. Detection of Bursaphelenchus Xylophilus, Causal Agent of Pine Wilt Disease on Pinus pinaster in Northwestern Spain.

Alvarez G., Etxebeste .I, Gallego D., David G., Bonifacio L., Jactel H., Sousa E \& Pajares JA.,2015. Optimization of traps for live trapping of Pine Wood Nematode vector Monochamus galloprovincialis. Journal of Applied Entomology 139:618-626.

Evans JS., Hudak AT., Faux R \& Smith AMS.,2009. Discrete return lidar in natural resources: Recommendations for project planning, data processing, and deliverables. Remote Sensing 1:776-794.

Ibeas F., Gallego D., Díez JJ \& Pajares Alonso JA .,2007. An operative kairomonal lure for managing pine sawyer beetle Monochamus galloprovincialis (Coleoptera: Cerymbycidae). Journal of Applied Entomology 131:13-20.

Mas i Gisbert H .,2015. Fenología y capacidad de dispersión de Monochamus galloprovincialis (Olivier 1795) en la península ibérica.

Mota M., Braasch H., Bravo M., Penas AC., Burgermeister W., Metge K \& Sousa E .,1999. First report of Bursaphelenchus xylophilus in Portugal and in Europe. Nematology $1: 727-734$.

Naves P., De Sousa EM \& Quartau JA .,2006. Feeding and oviposition preferences of Monochamus galloprovincialis for certain conifers under laboratory conditions. Entomologia Experimentalis et Applicata 120:99-104.

Pajares Alonso JA., Álvarez G., Ibeas F., Gallego D, Hall DR \& Farman DI .,2010. Identification and field activity of a male-produced aggregation pheromone in the pine sawyer beetle, Monochamus galloprovincialis. Journal of Chemical Ecology 36:570-583.

Pajares Alonso JA., Ibeas F., Díez JJ \& Gallego D .,2004. Attractive reponses by Monochamus galloprovincialis (Col., Cerambycidae) to host and bark bettle semiochemicals. Journal of Applied Entomology 128:633-638.

Robinet C., Roques A., Pan H., Fang G., Ye J., Zhang Y \& Sun J.,2009. Role of human-mediated dispersal in the spread of the pinewood nematode in China. PloS one 4:e4646.

Shibata E .,1986. Dispersal movement of the adult japanese Pine Sawyer, Monochamus al- 
ternatus Hope( Coleopter: cerambycidae) in a young pine forest. Applied Entomology and Zoology 21:184-186.

Sousa E., Bravo M., Pires J \& Naves P .,2001. Bursaphelenchus xylophilus (Nematoda; aphelenchoididae) associated with Monochamus galloprovincialis (Coleoptera; Cerambycidae) in Portugal. Nematology 3:89-91.

Togashi K .,1990. A field experiment on dispersal of newly emerged adults of Monochamus alternatus (Coleoptera: Cerambycidae). Researches on Population Ecology 32:1-13.

Yano S .,1913. Investigation on pine death in Nagasaki prefecture (in Japanese). Sanrin-Kouhou 4:1-14.

Yoshimura A., Kawasaki K., Takasu F., Togashi K., Futai K \& Shigesada N.,1999. Modeling the spread of pine wilt disease caused by nematodes with pine sawyers as vector. Eco$\log y$ 80:1691-1702.

Zamora P., Rodríguez V., Renedo F., Sanz AV., Domínguez JC., Escolar G., Miranda J., Álvarez B., González-Casas A., Mayor E., Dueñas M, Miravalles A., Navas A., Robertson L., Martín AB., 2015. First report of Bursaphelenchus xylophilus causing Pine Wilt Disease on Pinus radiata in Spain. Plant Disease http://dx.doi.org/10.1094/PDIS-03-15-0252PDN (accessed 10.5.15). 